\title{
Repeated fed-batch strategy and metabolomic analysis to achieve high docosahexaenoic acid productivity in Crypthecodinium cohnii
}

Liangsen Liu ${ }^{1,2,4 \dagger}$, Fangzhong Wang ${ }^{1,2,3 \dagger}$, Guangsheng Pei ${ }^{1,2,4}$, Jinyu Cui 1,2,4 , Jinjin Diao ${ }^{1,2,4}$, Mingming Lv²,4, Lei Chen ${ }^{1,2,4}$ and Weiwen Zhang ${ }^{1,2,3,4^{*}}$ (i)

\begin{abstract}
Background: Docosahexaenoic acid (DHA) is essential for human diet. However, high production cost of DHA using C. cohnii makes it currently less competitive commercially, which is mainly caused by low DHA productivity. In recent years, repeated fed-batch strategies have been evaluated for increasing the production of many fermentation products. The reduction in terms of stability of culture system was one of the major challenges for repeated fed-batch fermentation. However, the possible mechanisms responsible for the decreased stability of the culture system in the repeated fedbatch fermentation are so far less investigated, restricting the efforts to further improve the productivity. In this study, a repeated fed-batch strategy for DHA production using C. cohnii M-1-2 was evaluated to improve DHA productivity and reduce production cost, and then the underlying mechanisms related to the gradually decreased stability of the culture system in repeated fed-batch culture were explored through LC- and GC-MS metabolomic analyses.
\end{abstract}

Results: It was discovered that glucose concentration at $15-27 \mathrm{~g} / \mathrm{L}$ and $80 \%$ medium replacement ratio were suitable for the growth of $C$. cohnii M-1-2 during the repeated fed-batch culture. A four-cycle repeated fed-batch culture was successfully developed and assessed at the optimum cultivation parameters, resulting in increasing the total DHA productivity by $26.28 \%$ compared with the highest DHA productivity of $57.08 \mathrm{mg} / \mathrm{L} / \mathrm{h}$ reported using C. cohnii, including the time required for preparing seed culture and fermentor. In addition, LC- and GC-MS metabolomics analyses showed that the gradually decreased nitrogen utilization capacity, and down-regulated glycolysis and TCA cycle were correlated with the decreased stability of the culture system during the long-time repeated fed-batch culture. At last, some biomarkers, such as Pyr, Cit, OXA, FUM, L-tryptophan, L-threonine, L-leucine, serotonin, and 4-guanidinobutyric acid, correlated with the stability of culture system of C. cohnii M-1-2 were identified.

Conclusions: The study proved that repeated fed-batch cultivation was an efficient and energy-saving strategy for industrial production of DHA using C. cohnii, which could also be useful for cultivation of other microbes to improve productivity and reduce production cost. In addition, the mechanisms study at metabolite level can also be useful to further optimize production processes for C. cohnii and other microbes.

Keywords: Repeated fed-batch culture, Docosahexaenoic acid, Productivity, Metabolomic, Stability, Crypthecodinium cohnii

\footnotetext{
*Correspondence: wwzhang8@tju.edu.cn

†Liangsen Liu and Fangzhong Wang contributed equally to this work

1 Laboratory of Synthetic Microbiology, School of Chemical Engineering \&

Technology, Tianjin University, Tianjin 300072, People's Republic of China

Full list of author information is available at the end of the article
}

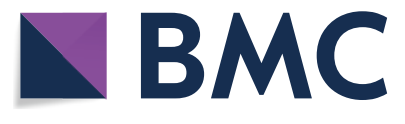

(c) The Author(s) 2020. This article is licensed under a Creative Commons Attribution 4.0 International License, which permits use, sharing, adaptation, distribution and reproduction in any medium or format, as long as you give appropriate credit to the original author(s) and the source, provide a link to the Creative Commons licence, and indicate if changes were made. The images or other third party material in this article are included in the article's Creative Commons licence, unless indicated otherwise in a credit line to the material. If material is not included in the article's Creative Commons licence and your intended use is not permitted by statutory regulation or exceeds the permitted use, you will need to obtain permission directly from the copyright holder. To view a copy of this licence, visit http://creativeco mmons.org/licenses/by/4.0/. The Creative Commons Public Domain Dedication waiver (http://creativecommons.org/publicdomain/ zero/1.0/) applies to the data made available in this article, unless otherwise stated in a credit line to the data. 


\section{Background}

Docosahexaenoic acid (DHA) that is essential for human diet, a long chain polyunsaturated fatty acids (PUFAs) of $\omega-3$ family, is an important structure component of human brain and retina [1]. Clinical studies showed that DHA also has positive effects on preventing some diseases, such as hypertension, atherosclerosis and some cancers [2]. The traditional source of DHA is fish oils. However, due to ocean pollution and sharp decrease of fish resources in recent decades, fermentation of heterotrophic microalgae has been proposed to be a promising alternative for DHA production [3, 4]. Crypthecodinium cohnii, a DHA-rich heterotrophic marine microalgae, has received great interest since it contains more than $50 \%$ lipid content in its dry cell weight (DCW) $[3,5]$; and the ratio of DHA in total fatty acids (TFAs) approaches $50 \%$ [5]; furthermore, it does not contain any eicosapentaenoic acid (EPA) that is not suitable for infant use [6]. In recent years, C. cohnii has been widely used for industrial production of DHA in many countries [3, 7].

One of major issues for industrial production of DHA using C. cohnii is the high production cost, which is caused by low DHA productivity. Significant efforts have been made in the past to improve the DHA productivity in C. cohnii by fed-batch cultures $[3,8]$. For example, $48 \mathrm{mg} / \mathrm{L} / \mathrm{h}$ and $53 \mathrm{mg} / \mathrm{L} / \mathrm{h}$ of DHA productivity were obtained using acetic acid and ethanol as the carbon source, respectively $[8,9]$. The DHA productivity was further elevated to $57.08 \mathrm{mg} / \mathrm{L} / \mathrm{h}$ using glucose as the carbon source [3]. However, for the purpose of the industrial production, elevation of DHA productivity by fed-batch culture alone may not be enough. According to recent studies $[10,11]$, repeated fed-batch strategy not only retains the advantages of fed-batch culture, such as relieving substrate inhibition, but also has many other distinctive characteristics. For example: (i) it saves the time and labor costs for seed preparation, cleaning and sterilization of the fermentor [10, 12]; (ii) it alleviates the inhibition of by-products, which typically accumulate to high contents during the stationary or late phases of batch and fed-batch fermentation [13]; (iii) it is beneficial to the stable downstream processes of product separation and purification [11]. In fact, in recent years repeated fed-batch strategies have been evaluated for increasing the production of many products $[11,14,15]$. For example, the production of the recombinant human serum albumin in yeast Pichia pastoris was enhanced by $47 \%$ through a repeated fed-batch fermentation strategy [11]. The cellulase productivity using $P$. oxalicum RE-10 was improved by $49.77 \%$ in a repeated fed-batch fermentation, compared with its batch fermentation [15]. The arachidonic acid (ARA) production period was shortened by $35 \%$ in a repeated fed-batch fermentation, compared to its fed-batch culture [14]. Therefore, these studies demonstrated that repeated fed-batch cultivation could be one of the promising measures to improve productivity and decrease fermentation cost of DHA production using C. cohnii.

As repeated fed-batch cultivation process typically lasts long time, the stability of culture system is an important factor $[14,16]$. However, the stability of culture system is usually decreased as the cycle number increased, which could lead to fermentation stop, affecting total productivity negatively. For example, a repeated fed-batch fermentation of lipid production using $R$. toruloides lasted only 2 cycles, and then the lipid productivity was significantly decreased in cycle 3 [17]; the lutein productivity started to decline in cycle 4 because DCW was decreased, during the repeated fed-batch culture of Desmodesmus sp. F51 [16]; the production of ARA by M. alpina stopped after four cycles, because the ARA percentage in TFAs was obviously decreased [14]. These early studies suggested that the reduction of the stability of culture system was one of the major challenges for repeated fed-batch fermentation. However, the possible mechanisms responsible for the decreased stability of culture system in the repeated fed-batch fermentation are so far less investigated, restricting the efforts to further improve the productivity.

Metabolomic analysis is able to provide a metabolitelevel representation of the phenotype for organisms due to its capability to detect a large array of small molecule metabolites qualitatively and quantitatively [18, 19]. In recent years, both liquid chromatography mass spectrometry (LC-MS) and gas chromatography mass spectrometry (GC-MS) based metabolomics have been widely applied to explore biology of various microalgae, including C. cohnii [20-22]. For example, the lipid accumulation mechanisms of $C$. cohnii responded to chemical modulators were explored using LC-MS based metabolomic analysis [21]; the glucose tolerance mechanisms of C. cohnii mutants obtained by adaptive laboratory evolution were analyzed by using GC-MS metabolomic analysis [22]; and the mechanisms of antioxidant on elevating lipid accumulation in C. cohnii were analyzed using integrated LC- and GC-MS metabolomic analyses [20]. Therefore, metabolomic analysis is considered to be a promising approach to study the changes in metabolites of C. cohnii during the long-time repeated fed-batch cultivation.

In this study, a repeated fed-batch fermentation strategy for efficient DHA production with C. cohnii was developed and evaluated by optimizing the parameters substrate concentration and medium replacement ratio. Metabolomic analysis was then used to explore the underlying mechanisms related to the reduced stability of 
culture system of $C$. cohnii during the repeated fed-batch fermentation process. This study provided an effective strategy for industrial production of DHA using C. cohnii, and new insights for maintaining the stability of culture system during the extended fermentation process.

\section{Results and discussion}

\section{Effects of glucose concentration on DHA production} in repeated fed-batch cultivation

Our previous study showed that the specific growth rate and specific glucose consumption rate of C. cohnii M-1-2 were both decreased and maintained at a low rate after $120 \mathrm{~h}$ in fed-batch culture with initial inoculated volume at $10 \%(v / v)$ [3]. Therefore, the fermentation broth was initially replaced at $120 \mathrm{~h}$ of each cycle with replacement ratio at $90 \%(v / v)$. The concentration of carbon sources plays an important role in microbial metabolism, including C. cohnii $[3,8,9,14]$. Therefore, the effect of glucose maintenance concentration on DHA production was first investigated in repeated fed-batch cultivation of C. cohnii M-1-2 (Fig. 1 and Table 1). The results showed that, for glucose concentration at 5-15 g/L, C. cohnii M-1-2 entered into stationary growth phase at $96 \mathrm{~h}$ in each cycle, and achieved the lowest DCW and DHA productivity of each cycle; for glucose concentration at 27-45 g/L, C. cohnii M-1-2 also entered into stationary growth phase at $96 \mathrm{~h}$ of each cycle; for glucose concentration at 15-27 g/L, C. cohnii M-1-2 kept growing and did not enter into stationary growth phase in both cycles during $120 \mathrm{~h}$, and eventually achieved the highest DCW and DHA productivity in both cycles (Table 1 ). In addition, the DHA yield on glucose at $15-27 \mathrm{~g} / \mathrm{L}$ was 47.61 and $40.42 \mathrm{mg} / \mathrm{g}$ for the two cycles, respectively, which was higher than the 39.92 and $34.15 \mathrm{mg} / \mathrm{g}$ on glucose at 5-15 g/L of the same two cycles. The DHA yield on glucose at $27-45 \mathrm{~g} / \mathrm{L}$ was $39.16 \mathrm{mg} / \mathrm{g}$ in cycle 2, which was higher than $33.08 \mathrm{mg} / \mathrm{g}$ in cycle 1 . Together, the results demonstrated that glucose concentration at $15-27 \mathrm{~g} / \mathrm{L}$ was relatively suitable for the growth of $C$. cohnii M-1-2 during the repeated fed-batch cultivation.

As mentioned above, glucose concentration maintained at $15-27 \mathrm{~g} / \mathrm{L}$ was more suitable for $C$. cohnii M-1-2 growth in comparison with that at $27-45 \mathrm{~g} / \mathrm{L}$, consistent with the previous reports that high glucose concentration inhibited the growth of C. cohnii ATCC 30556 or 30772 in batch culture [23, 24], in which the highest specific growth rate and DCW were achieved at an initial glucose concentration $20 \mathrm{~g} / \mathrm{L}$ of C. cohnii ATCC 30556 [23], and the initial growth rate of C. cohnii ATCC 30772 was reduced when the initial glucose concentration was above $25 \mathrm{~g} / \mathrm{L}$ [24]. The glucose inhibition on growth was also observed in other lipid producing microbes [17]. For example, the DCW of $R$. toruloides $\mathrm{Y} 4$ were increased by $37.8 \%$ at $5 \mathrm{~g} / \mathrm{L}$ of glucose concentration, compared with glucose concentration maintained at $30 \mathrm{~g} / \mathrm{L}$ [17]. High glucose concentration of $15-27$ and $27-45$ g/L was found to inhibit the DHA synthesis significantly in cycle 2 compared with that in cycle 1 (Fig. 3a). The inhibition to DHA synthesis by high glucose was also found in other C. cohnii studies [22, 23]. For example, the DHA percentage of TFAs in C. cohnii ATCC 30556 was reduced to $44.7 \%$ from $53.4 \%$, when glucose concentration was increased to $20 \mathrm{~g} / \mathrm{L}$ from $5 \mathrm{~g} / \mathrm{L}$ [23]. The total lipid in DCW and DHA percentage of TFAs in C. cohnii ATCC 30556 were all gradually decreased with the increase of glucose concentration from 9 to $54 \mathrm{~g} / \mathrm{L}$ [22]. One possible reason of high glucose inhibition might be the increased osmotic pressure of the culture medium, which results in more energy consumption required for cellular maintenance [25]. A higher initial number of cells was found to increase the stress adaptation of Escherichia coli [26]. The final DCW of cycle 2 with glucose concentration at $27-45 \mathrm{~g} / \mathrm{L}$ was increased by $17.09 \%$, compared with that of cycle 1 with glucose concentration at $27-45 \mathrm{~g} / \mathrm{L}$, which might be due to that the initial number of cells in the cycle 2 of $27-45 \mathrm{~g} / \mathrm{L}$ was much larger than that in the cycle 1 of $27-45 \mathrm{~g} / \mathrm{L}$, resulting in enhancing the resistance of C. cohnii M-1-2 to high glucose inhibition.

\section{Effects of medium replacement ratio on DHA production in repeated fed-batch cultivation}

Medium replacement ratio not only affects the composition of the fermentation broth, but also affects the inoculated cell number. Therefore, the medium replacement ratio was further optimized (Table 2). The results showed that DCW of $80 \%$ medium replacement ratio was increased by $11.15 \%$ and $5.21 \%$, compared with $70 \%$ and 90\% medium replacement ratio, respectively. The DHA content in DCW $(w / w)$ of $80 \%$ medium replacement ratio was elevated by $12.64 \%$ in comparison with $70 \%$ medium replacement ratio, but it remained at the similar level with $90 \%$ medium replacement ratio (Fig. 3b). The DHA productivity was the highest at $80 \%$ medium replacement ratio, which was $25.33 \%$ and $5.70 \%$ higher than $70 \%$ and 90\% medium replacement ratios, respectively. Therefore, $80 \%$ medium replacement ratio was selected for the repeated fed-batch cultivation of C. cohnii M-1-2.

The DCW, DHA content in DCW $(w / w)$ and DHA productivity of cycle 2 were all decreased at $70 \%$ medium replacement ratio compared with those at $80 \%$ medium replace ratio of cycle 2 (Table 2 and Fig. $3 \mathrm{~b}$ ). It was previously known that $C$. cohnii produced a large amount of extracellular polysaccharides at the late stage of the fermentation process, which increased the viscosity of fermentation broth and reduced the efficiency of oxygen transfer, leading to a low DHA production [27]. 

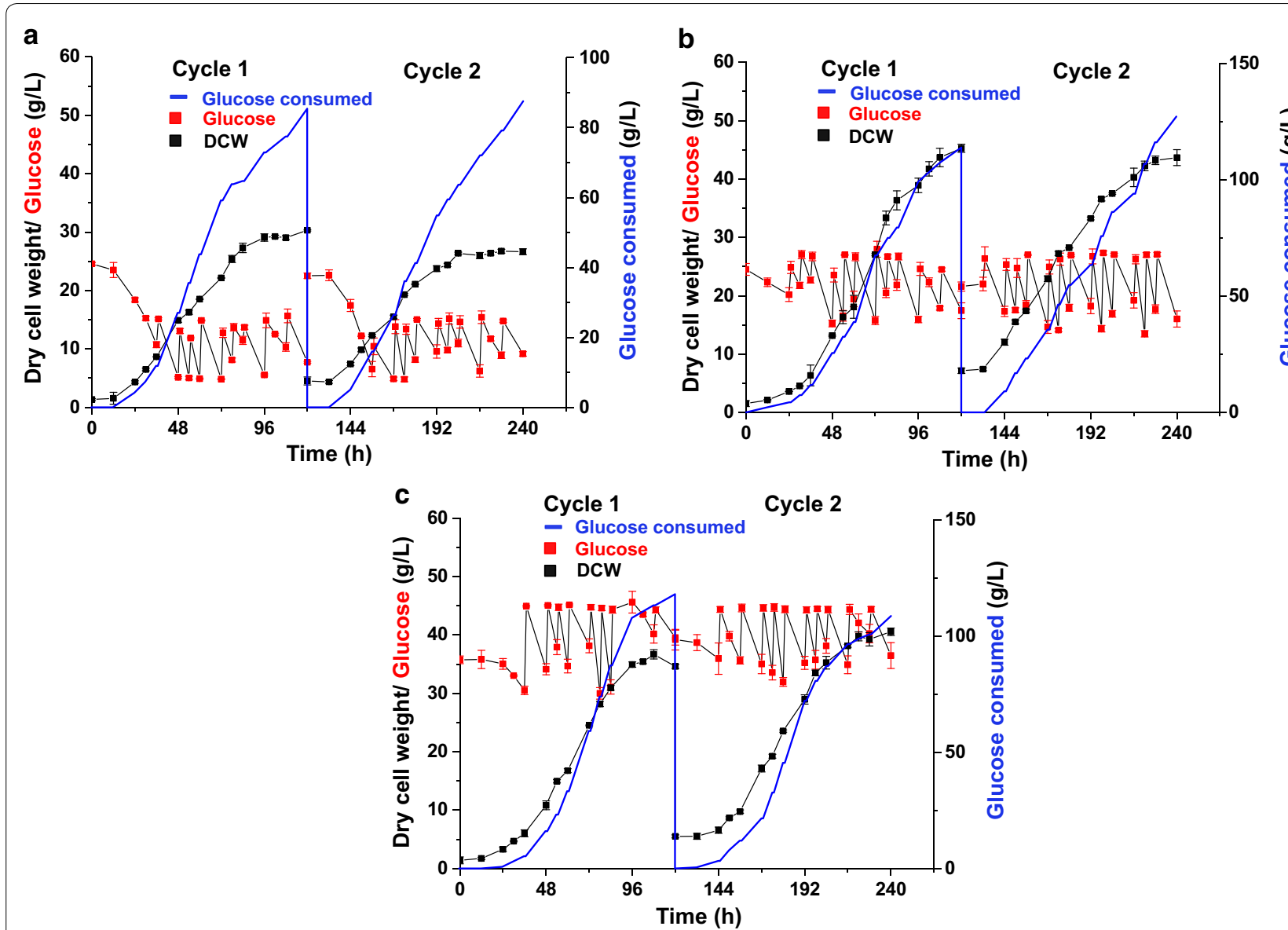

Fig. 1 Repeated fed-batch cultivation of two cycles under different glucose concentrations at $25^{\circ} \mathrm{C}$ and $\mathrm{pH}$ 6.5. a Repeated fed-batch culture with initial glucose monohydrate concentration $27 \mathrm{~g} / \mathrm{L}$ and maintenance concentration 5-15 g/L of glucose; b Repeated fed-batch culture with initial glucose monohydrate concentration $27 \mathrm{~g} / \mathrm{L}$ and maintenance concentration 15-27 g/L of glucose; c Repeated fed-batch culture with initial glucose monohydrate concentration $45 \mathrm{~g} / \mathrm{L}$ and maintenance concentration $27-45 \mathrm{~g} / \mathrm{L}$ of glucose

Table 1 Comparison of DHA production at the end of each cycle using different glucose concentrations

\begin{tabular}{|c|c|c|c|c|c|}
\hline Glucose Conc. (g/L) & Cycle & Fermentation time (h) & Dry cell weight (g/L) & DHA (g/L) & $\begin{array}{l}\text { DHA } \\
\text { productivitya } \\
\text { (mg/L/h) }\end{array}$ \\
\hline $5.00-15.00$ & Cycle 1 & 120 & $30.38 \pm 0.12$ & $3.41 \pm 0.03$ & $28.44 \pm 0.24$ \\
\hline $5.00-15.00$ & Cycle 2 & 120 & $26.72 \pm 0.49$ & $2.99 \pm 0.01$ & $24.93 \pm 0.10$ \\
\hline $15.00-27.00$ & Cycle 1 & 120 & $45.17 \pm 0.71$ & $5.42 \pm 0.02$ & $45.14 \pm 0.15$ \\
\hline $15.00-27.00$ & Cycle 2 & 120 & $43.57 \pm 1.37$ & $5.14 \pm 0.04$ & $42.84 \pm 0.30$ \\
\hline $27.00-45.00$ & Cycle 1 & 120 & $34.69 \pm 0.35$ & $3.90 \pm 0.01$ & $32.54 \pm 0.12$ \\
\hline $27.00-45.00$ & Cycle 2 & 120 & $40.62 \pm 0.63$ & $4.25 \pm 0.03$ & $35.40 \pm 0.25$ \\
\hline
\end{tabular}

${ }^{a}$ DHA productivity of each cycle (the DHA produced at the end of each cycle/the time of each cycle operation)

Therefore, we speculated that extra-metabolites or byproducts secreted by $C$. cohnii and possible accumulation of unspent medium components might affect the growth and DHA synthesis, resulting in inhibition of the growth and DHA synthesis at 70\% medium replacement ratio. When the replacement ratio was maintained at $80 \%$, the inhibition was efficiently relieved [28]. Medium replacement ratio was also found as an important parameter in several other studies in repeated fed-batch culture $[12,15]$. For example, the $80 \%$ medium replacement 
Table 2 Comparison of DHA production by repeated fed-batch culture using different medium replacement ratios

\begin{tabular}{|c|c|c|c|c|c|}
\hline Replacement ratio (\%) & Cycle & Fermentation time (h) & Dry cell weight (g/L) & $\mathrm{DHA}(\mathrm{g} / \mathrm{L})$ & $\begin{array}{l}\text { DHA } \\
\text { productivity } \\
\text { (mg/L/h) }\end{array}$ \\
\hline $90^{\mathrm{a}}$ & Cycle 2 & 120 & $43.57 \pm 1.37$ & $5.14 \pm 0.04$ & $42.84 \pm 0.30$ \\
\hline 80 & Cycle 2 & 120 & $45.84 \pm 0.52$ & $5.43 \pm 0.02$ & $45.28 \pm 0.13$ \\
\hline 70 & Cycle 2 & 120 & $41.24 \pm 0.78$ & $4.34 \pm 0.15$ & $36.13 \pm 1.29$ \\
\hline
\end{tabular}

a The results were from Table 1

ratio was found to be the optimized culture condition for Arthrospira (Spirulina) platensis in the repeated fed-batch culture compared with $50 \%$ or $95 \%$ [29]. The DHA productivity of Schizochytrium sp. was increased by $27.84 \%$ at $80 \%$ medium replacement ratio compared with $95 \%$ in repeated fed-batch culture [12]. However, $50 \%$ medium replacement ratio was found to be the most suitable for the production of cellulase by Penicillium oxalicum RE-10 [15], and 50\% medium replacement ratio was also used to achieve the best production of xylitol by Candida magnoliae TISTR 5663 [30]. One of the possible reasons for the difference in the optimal medium replacement ratio might be related to the types of microbes and products $[15,31]$.

\section{DHA production in repeated fed-batch cultivation} with multiple cycles

After optimizing glucose concentration and medium replacement ratio in repeated fed-batch culture with two cycles, efforts were made to achieve more cycles (Fig. 2). For the repeated fed-batch culture with multiple cycles, the initial inoculation volume was set as $20 \%(v / v)$ and the medium replacement ratio was $80 \%(v / v)$ with glucose concentration maintained at $15-27 \mathrm{~g} / \mathrm{L}$, respectively. The results showed that the DCW were at the similar level of both cycle 2 and cycle 3 compared with that of cycle 1 , but it was reduced significantly by $4.36 \%$ of cycle 4 compared with that of cycle 1 , as demonstrated by a statistical analysis (Table 3). The DHA content in DCW $(w / w)$ remained the similar level through all four cultivation cycles (Fig. 3c). The results suggested that growth rather than the DHA synthesis of C. cohnii M-1-2 started to be inhibited at cycle 4 (Table 3 and Fig. 3c). Moreover, the DHA yield on glucose of each cycle were $47.72,45.80$, 44.82 , and $39.70 \mathrm{mg} / \mathrm{g}$ respectively with a $16.81 \%$ drop between cycle 1 and cycle 4 , which was due to a $16.67 \%$ drop of DCW yield on glucose between cycle 1 and cycle 4. In addition, the color of fermentation broth gradually

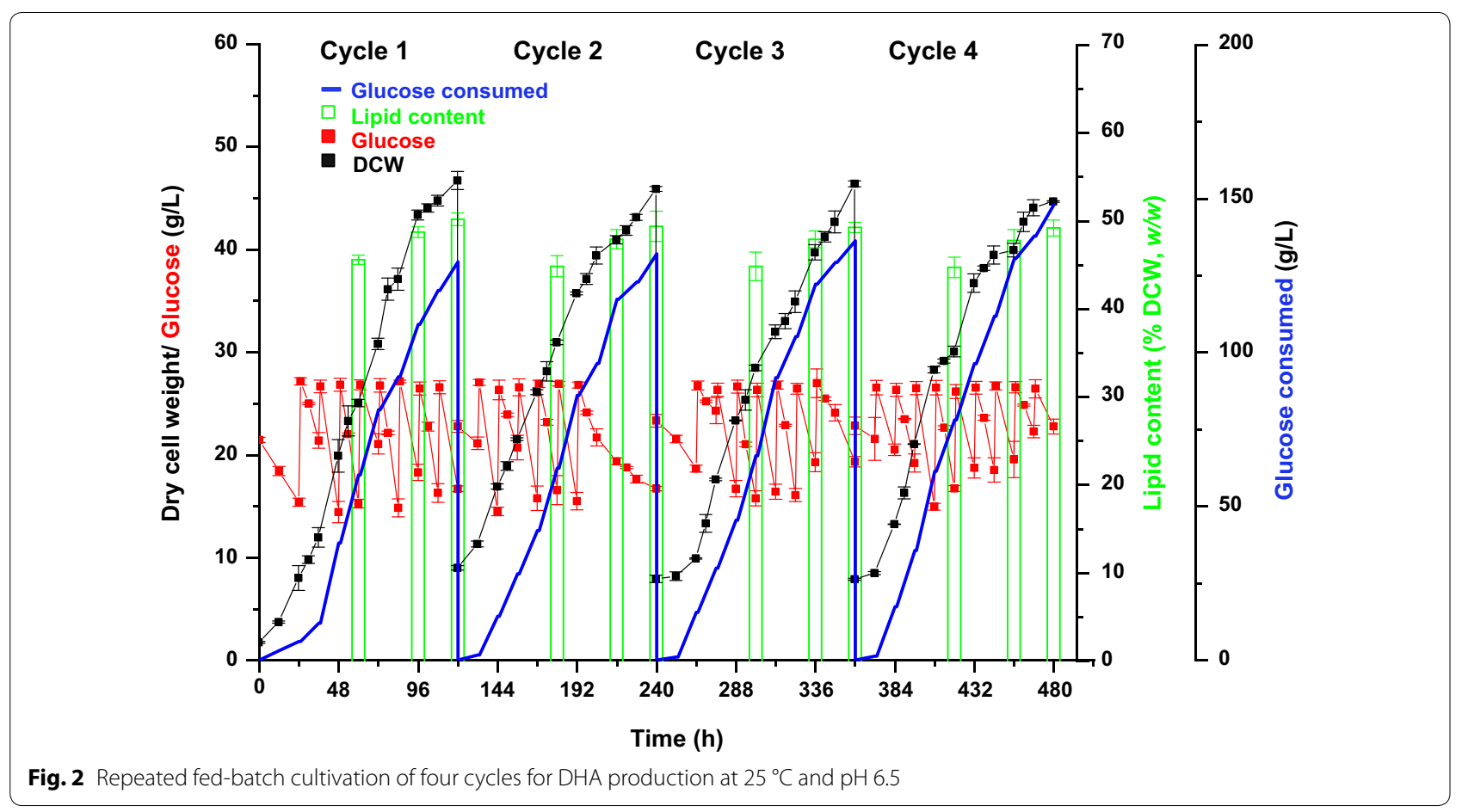




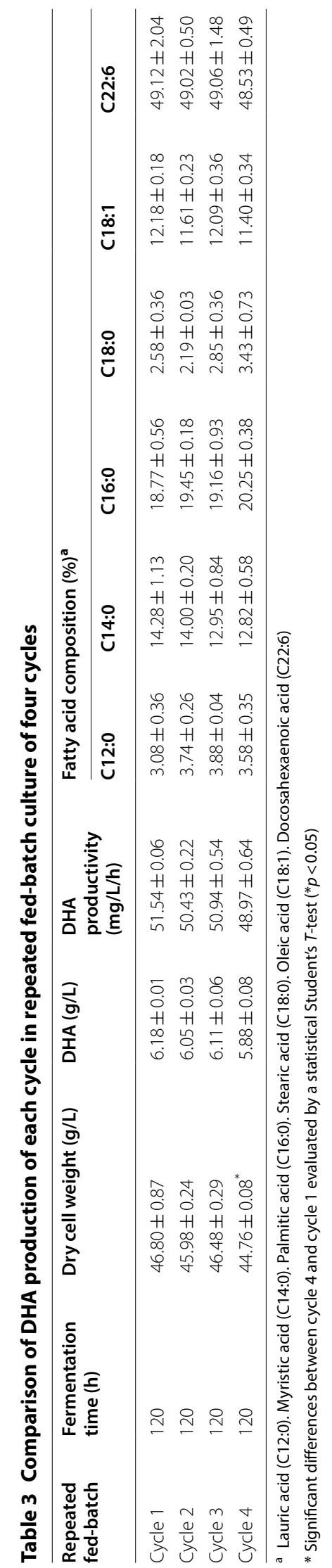


became dark mainly due to cell mass with the cycle number increased (data not shown), which also suggested cell state might be changed [14]. Therefore, the repeated fed-batch culture of C. cohnii M-1-2 was stopped after cycle 4 . Table 4 showed the DHA productivity by C. cohnii using different fermentation strategies. The analysis showed that only two DHA productivities using C. cohnii $[3,9]$, were slightly higher than each individual cycle of the repeated fed-batch culture, so the DHA productivity of each individual cycle in this study was all maintained at a relatively high level compared with other fermentation strategies. Furthermore, the total productivity of repeated fed-batch culture with 4 cycles was increased by $26.28 \%$ compared with the reported highest DHA productivity of $57.08 \mathrm{mg} / \mathrm{L} / \mathrm{h}$ in C. cohnii considering the time for preparing seed culture and fermentor, suggesting its application advantages [3, 14]. What's more, the lipid content in DCW $(w / w)$ and fatty acids profiles both showed no changes, which suggested that the quality of our products was stable during the repeated fed-batch with 4 cycles (Fig. 2 and Table 3). Together, a repeated fed-batch strategy to achieve high DHA productivity in C. cohnii was successfully established.

Seed culture is important for the final DCW and DHA productivity of $C$. cohnii, but it took much time and efforts to prepare seed culture for fed-batch fermentation $[3,8,9,24]$. Repeated fed-batch fermentation only needs inoculation once and saves time on cleaning and sterilization of fermenter, greatly improved the productivity [14]. In addition, the costs of staffs, seed raw materials, and
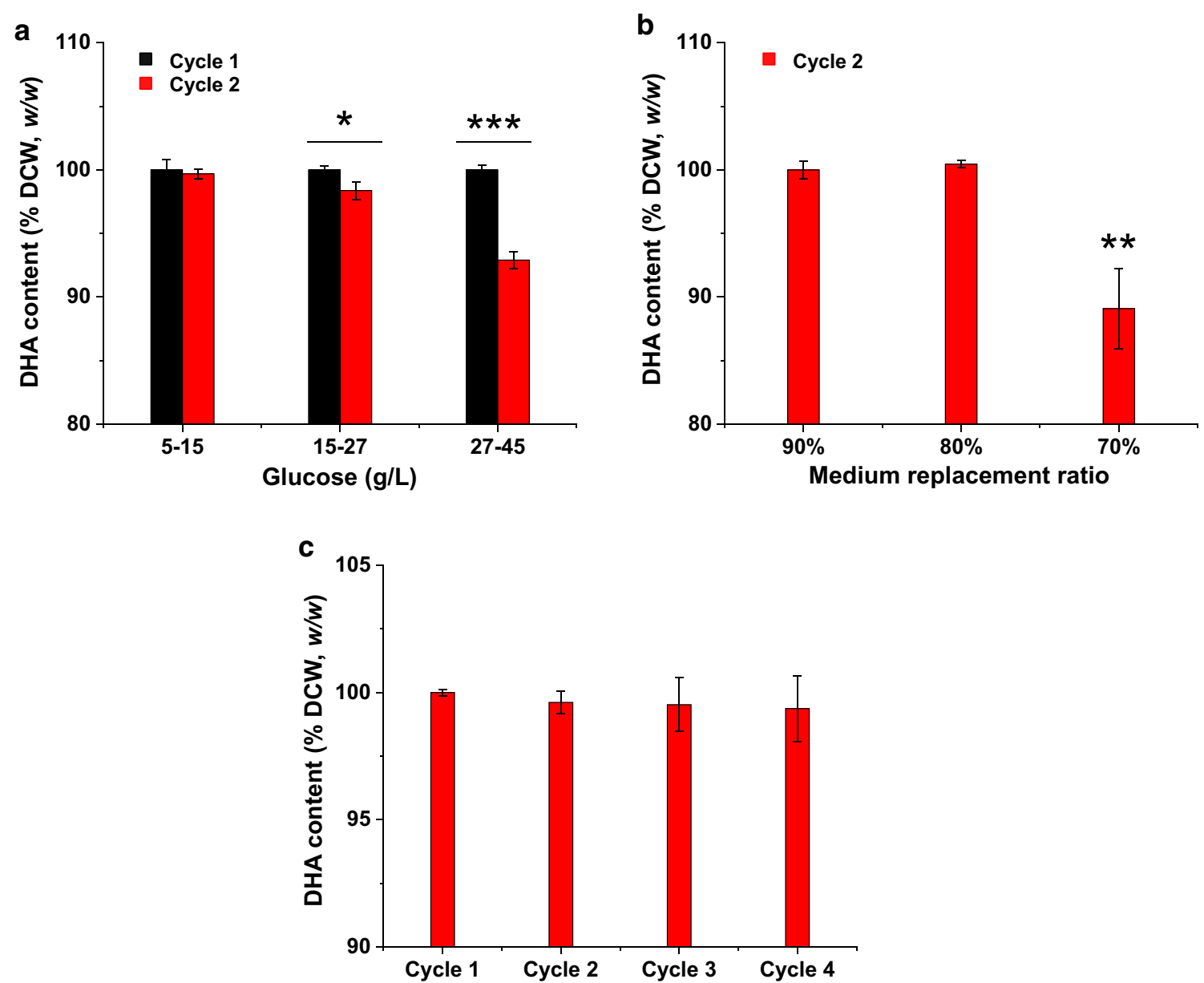

Fig. 3 DHA content (\% DCW, $/ W$ ) in repeated fed-batch cultivation of two and four cycles. a DHA content of two cycles under different glucose concentrations. The DHA content in cycle 1 was set as 100\% under different glucose concentrations respectively, and the percentage changes in cycle 2 were shown; $\mathbf{b}$ DHA content of two cycles under different medium replacement ratios. The DHA content in cycle 2 under medium replacement ratio of $90 \%$ was set as 100\%, and the percentage changes in cycle 2 under medium replacement ratio of $80 \%$ and $70 \%$ were shown; c DHA content of four cycles. The DHA content in cycle 1 was set as 100\%, and the percentage changes in other cycles were shown. Asterisks indicated significant differences, as evaluated by a statistical Student's $T$-test $\left({ }^{*} p<0.05,{ }^{* *} p<0.01,{ }^{* *} p<0.001\right)$ 
Table 4 Comparison of DHA productivity using C. cohnii by different culture strategies

\begin{tabular}{|c|c|c|c|c|c|}
\hline Culture strategy & Crypthecodinium cohnii & Carbon source & $\begin{array}{l}\text { Fermentation time } \\
\text { (h) }\end{array}$ & $\begin{array}{l}\text { DHA productivity } \\
\text { (mg/L/h) }\end{array}$ & References \\
\hline Fed-batch & CCMP 316 & Glucose & 135 & 9.78 & {$[48]$} \\
\hline Batch & ATCC 30772 & Glucose & 91 & 17.58 & [24] \\
\hline Fed-batch & CCMP 316 & Carob pulp & 100 & 19.00 & [49] \\
\hline Fed-batch & ATCC 30556 & Date syrup & 240 & 23.54 & {$[7]$} \\
\hline Fed-batch & ATCC 30772 & Acetic acid & 400 & 47.50 & [8] \\
\hline Fed-batch & ATCC 30772 & Ethanol & 220 & 53.18 & {$[9]$} \\
\hline Fed-batch & $M-1-2$ & Glucose & 168 & 57.08 & {$[3]$} \\
\hline Repeated fed-batch cycle 1 & $M-1-2$ & Glucose & 120 & 51.54 & This study \\
\hline Repeated fed-batch cycle 2 & $M-1-2$ & Glucose & 120 & 50.43 & This study \\
\hline Repeated fed-batch cycle 3 & $M-1-2$ & Glucose & 120 & 50.94 & This study \\
\hline Repeated fed-batch cycle 4 & $M-1-2$ & Glucose & 120 & 48.97 & This study \\
\hline
\end{tabular}

the energy consumption for cleaning and sterilization of fermenter could be apparently reduced [12]. Therefore, repeated fed-batch culture was a good option to reduce the cost of DHA production in C. cohnii, especially for large-scale industrial production. Repeated fed-batch culture has also been used successfully in the culture of many other microorganisms, such as P. oxalicum RE-10 and C. parapsilosis $[15,32]$.

As mentioned above (Table 3), the DCW and DCW yield on glucose of C. cohnii M-1-2 were both decreased significantly at the cycle 4 compared with those of cycle 1 , and the color of fermentation broth was gradually changed,resulting in the fermentation stopping after cycle 4 . Therefore, the stability of the culture system seemed gradually decreased as cycle number increased. It has been demonstrated that $70 \%$ replacement ratio can result in the inhibition to C. cohnii M-1-2 (Table 2). Therefore, it was reasonable to speculate the possible accumulation of inhibition compounds in the medium either from the growth medium or secreted by the cells, and the possible changed cell stability might be the reasons for the changes about the stability of culture system as cycle number increased and then the DCW of C. cohnii M-1-2 would be affected negatively [31, 33]. Similar phenomenon was also observed in other studies during the repeated fed-batch culture [14, 33].

\section{LC-MS based metabolomic analysis of the repeated fed-batch culture with 4 cycles}

LC-MS metabolomic analysis aiming mainly at unstable metabolites of central carbohydrate and energy metabolism [20] have been well established to probe the physiological change of C. cohnii M-1-2 [20, 21]. Cell samples collected at 60 and $96 \mathrm{~h}$ of each cycle, which were both in the linear growth phases, were subjected to LC-MS based metabolic analysis to explore possible mechanisms related to the changes about the stability of culture system during repeated fed-batch culture of C. cohnii M-12. As shown in Fig. 4, the content of metabolites was changed gradually as the cycle number increased, including: (i) intracellular abundances of amino acids, such as valine, leucine, glutamic acid, lysine, and phenylalanine at $60 \mathrm{~h}$, and glycine, leucine, glutamic acid, lysine, and phenylalanine at $96 \mathrm{~h}$, were constantly up-regulated as the cycle progressed (Fig. 4). C. cohnii M-1-2 was known to accumulate large amounts of amino acids under high nitrogen condition [3]. Continuous up-regulation of these amino acids suggested that the nitrogen utilization capacity of $C$. cohnii M-1-2 might be constantly decreased as cycle number increased; (ii) the continuous up-regulation of PEP, 3-PG, 1,3-PG, GAP, F6P, and FBP at $96 \mathrm{~h}$, and the continuous up-regulation of GAP at $60 \mathrm{~h}$ was observed. However, Pyr at 60 and $96 \mathrm{~h}$ was both gradually down-regulated, suggesting that the metabolic flux from glycolysis pathway was gradually decreased as cycle number increased; (iii) the continuous down-regulation of Cit, OXA, and FUM at $60 \mathrm{~h}$, and the continuous down-regulation of Cit, OXA, FUM, and AKG at $96 \mathrm{~h}$ suggested that tricarboxylic acid (TCA) cycle might be gradually attenuated as the cycle number increased. Therefore, the up-regulation of amino acids might consume the carbon skeletons of a large number of organic acids from TCA cycle, which might lead to the down-regulation of TCA cycle, reducing the cell activity of C. cohnii M-1-2 [3, 34]. Down-regulated TCA cycle has also been found in other aging organisms and cells, such as Drosophila melanogaster and testicular [35, 36]. It was interesting to find that the changes of metabolites above had two distinct characteristics. One was that the changes between the two adjacent cycles were small, but it was large between the cycle 1 and cycle 4 . Another was that the change direction of the same metabolites was 

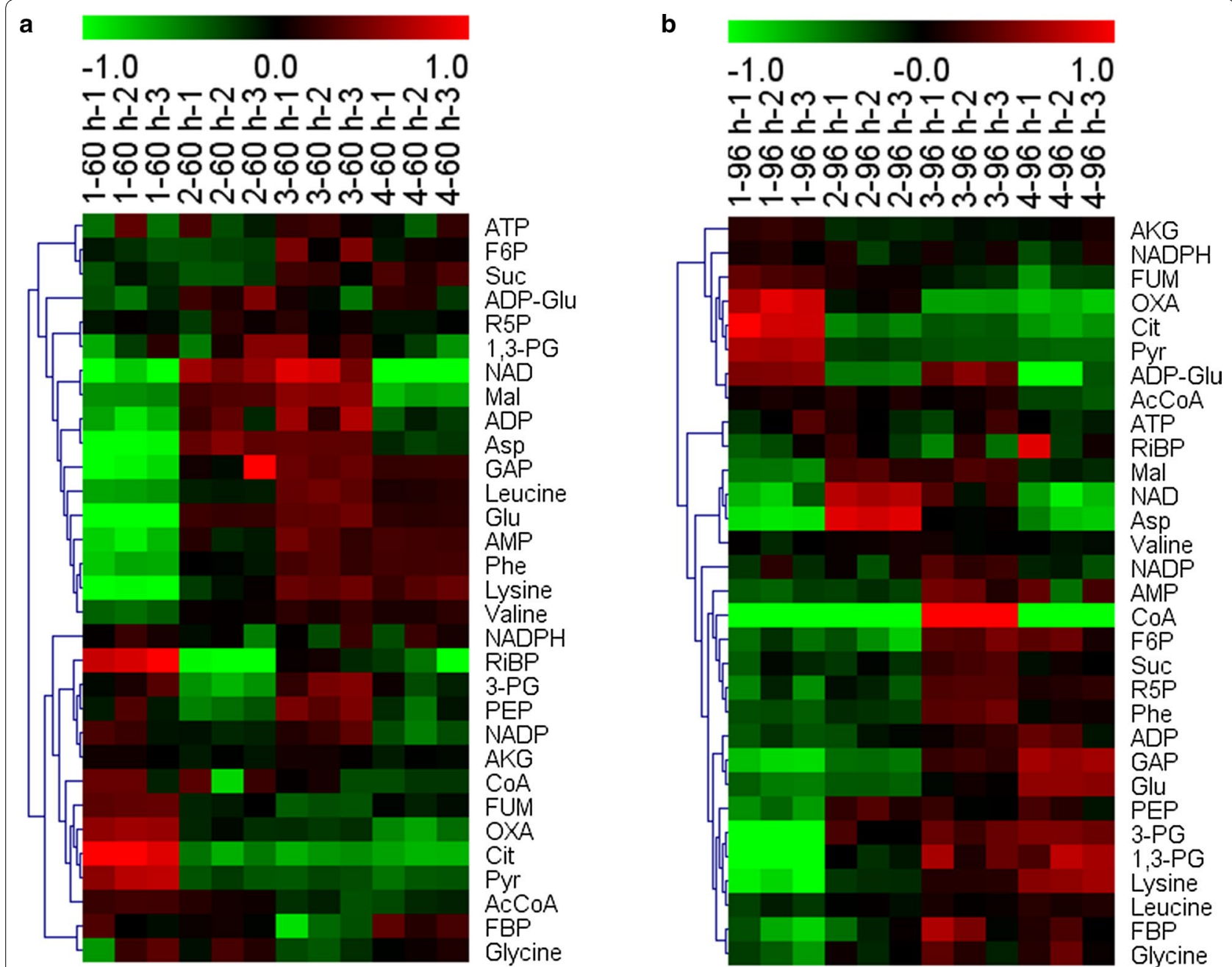

Fig. 4 Heatmaps of LC-MS metabolomic profiles of repeated fed-batch cultivation with 4 cycles. $\mathbf{a} 60 \mathrm{~h} ; \mathbf{b} 96 \mathrm{~h}$. The first number represents the number of cycles and the third number represents biological replicates of 1-60 h-1

consistent among different cycles. The possible reasons that only the DCW of cycle 4 decreased compared with that of cycle 1 might be that certain degrees of changes were required to affect the stability of culture system negatively. In summary, these analyses suggested that the gradually decreased nitrogen utilization capacity, the continuous down-regulated glycolysis and TCA cycle might be the key factors that affected the stability of culture system of C. cohnii M-1-2 during the repeated fedbatch culture with multiple cycles.

\section{GC-MS based metabolomic analysis of the repeated fed-batch culture with 4 cycles}

GC-MS based metabolomic analysis mainly aims at relatively stable metabolites with broader metabolite coverage [20]. In order to further study the mechanisms of attenuated stability of culture system in C. cohnii M-1-2 during the repeated fed-batch culture with 4 cycles, GCMS based metabolomic analysis was carried out. Samples were collected at 60 and $96 \mathrm{~h}$ of each cycle during the repeated fed-batch culture. A total of 101 intracellular metabolites were identified in all samples. PCA was used to analyze the overall distribution of the 101 metabolites (Fig. 5). Five biological replicates of each sample gathered well, which indicated that they had good reproducibility. The gradually increased differences at metabolite level for the same time point of different cycles suggested that the changes in cell states were gradually accumulated with the cycle number increased. However, the changes of the relative content of metabolites at the same time point between cycle 1 and 4 were not particularly large, which was consistent with that the DCW change between cycle 1 and 4 of $4.36 \%$. 


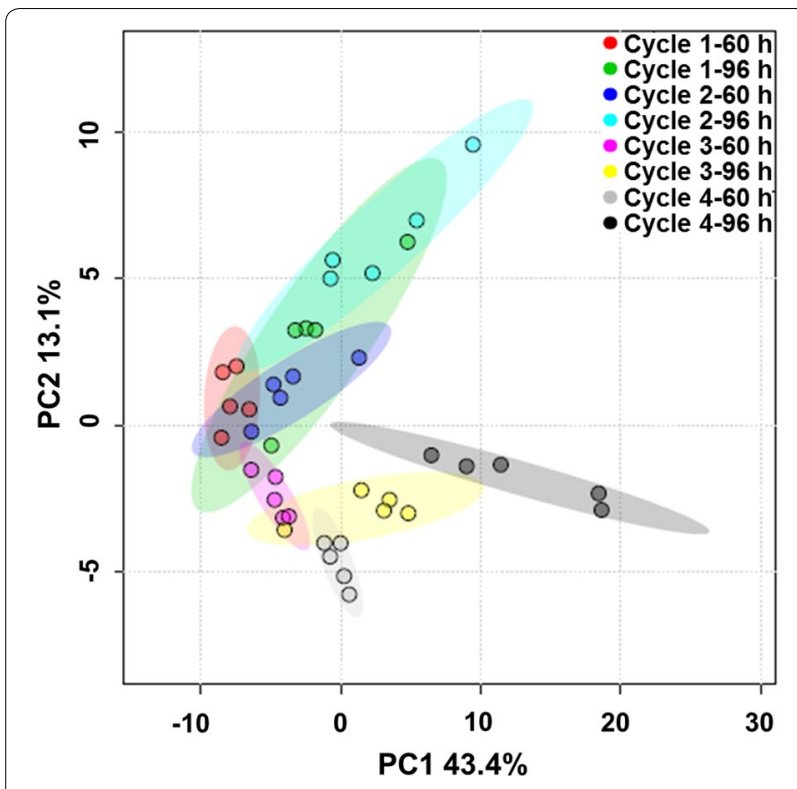

Fig. 5 PCA of GC-MS metabolomic profiles of repeated fed-batch cultivation with 4 cycles. The first and second number labeled on top right legends (e.g. cycle 1-60 h) represent the number of cycle and cultivation time, respectively

WGCNA is a powerful tool for analyzing omics data [37]. Hub metabolites are highly interconnected metabolites in biological interaction networks, which are considered to have important biological significance $[37,38]$. WGCNA was used to identify hub metabolites related to the stability of culture system during the long-term fermentation (Fig. 6). The WGCNA of the GC-MS metabolomic data presented only three modules, suggesting that the changes of metabolites concentration were limited, which was consistent with the PCA results (Fig. 5 and 6a). Using a cutoff of the correlation coefficient $r>0.6$ (statistical confidence $p<0.05$ ), the blue module $\left(r=0.7\right.$, correlation $p$-value $\left.=5 \mathrm{e}^{-07}\right)$ was found to be positively associated with sampling time points (i.e. 60 and $96 \mathrm{~h}$ ), while the turquoise module $\left(r=0.65\right.$, correlation $p$-value $\left.=5 \mathrm{e}^{-06}\right)$ was positively associated with the cycle number. These two modules were further subjected to metabolic network topology analysis and identification of hub metabolites (Fig. 6b, c). Using a cutoff of hub metabolites with connectivity at least 5, allo-inositol, L-tyrosine, and glycerol 1-phosphate located in blue module were found to be positively associated with sampling time points, while L-tryptophan, L-threonine, L-leucine, serotonin, and 4-guanidinobutyric acid located in turquoise module were positively associated with the cycle number, respectively.
Glycerol 1-phosphate is the precursor for triacylglycerol that is the major lipid storage compound in C. cohnii [39]. Therefore, association of glycerol 1-phosphate with sampling time points suggested the content of glycerol 1-phosphate was increased at $96 \mathrm{~h}$ compared to that at $60 \mathrm{~h}$, which was consistent with the fact that the lipid content at $96 \mathrm{~h}$ was higher than at $60 \mathrm{~h}$ (Fig. 2). It was also consistent with previous findings that the lipid content (\% DCW, $w / w)$ of $C$. cohnii was increased with fermentation progressing $[9,40]$.

The metabolites located at the turquoise module were positively associated with the stability of culture system. The LC-MS based metabolomic analysis showed that PEP was gradually up-regulated at $96 \mathrm{~h}$ as the cycle number increased. GC-MS based metabolomic analysis showed L-tryptophan and serotonin were gradually up-regulated as the cycle number increased, suggesting that PEP might be used to synthesize L-tryptophan and serotonin. Tryptophan was also found to be up-regulated under different nitrogen supply conditions for several microbes including C. cohnii $[3,41]$. For example, the tryptophan was up-regulated under nitrogen excess in $C$. cohnii [3]. However, it is well known that the mechanism involved in tryptophan synthesis is complex and further study is still needed for better understanding the details related to the stability of culture system. In addition to the gradually changed metabolic network, C. cohnii M-1-2 might regulate cell metabolism through L-tryptophan, which have been reported to regulate cell metabolism in bacterial cells [42]. For example, tryptophan content in cell was negatively correlated with the growth rate of bacteria [42]. The up-regulation of L-threonine and L-leucine led to further decrease of carbon flow from TCA and glycolytic pathways. Finally, these identified metabolites by LC- and GC-MS could also be used as biomarkers representing the stability of culture system of C. cohnii. The identification of the relevant biomarkers provided useful information for monitoring the stability of culture system during the repeated fed-batch culture, as well as improving the stability of culture system of $C$. cohnii by genetic engineering in the future [43].

\section{Conclusions}

Herein, glucose concentration at $15-27 \mathrm{~g} / \mathrm{L}$ and $80 \%$ medium replacement ratio were found to be suitable for the growth of C. cohnii M-1-2 during the repeated fedbatch cultivation. A 4-cycle repeated fed-batch cultivation strategy for DHA production using C. cohnii was first successfully established and the total DHA productivity was increased by $26.28 \%$ compared with the highest DHA productivity of $57.08 \mathrm{mg} / \mathrm{L} / \mathrm{h}$ reported using $C$. cohnii, taking into consideration of the time for preparing seed culture and fermentor. In addition, gradually 


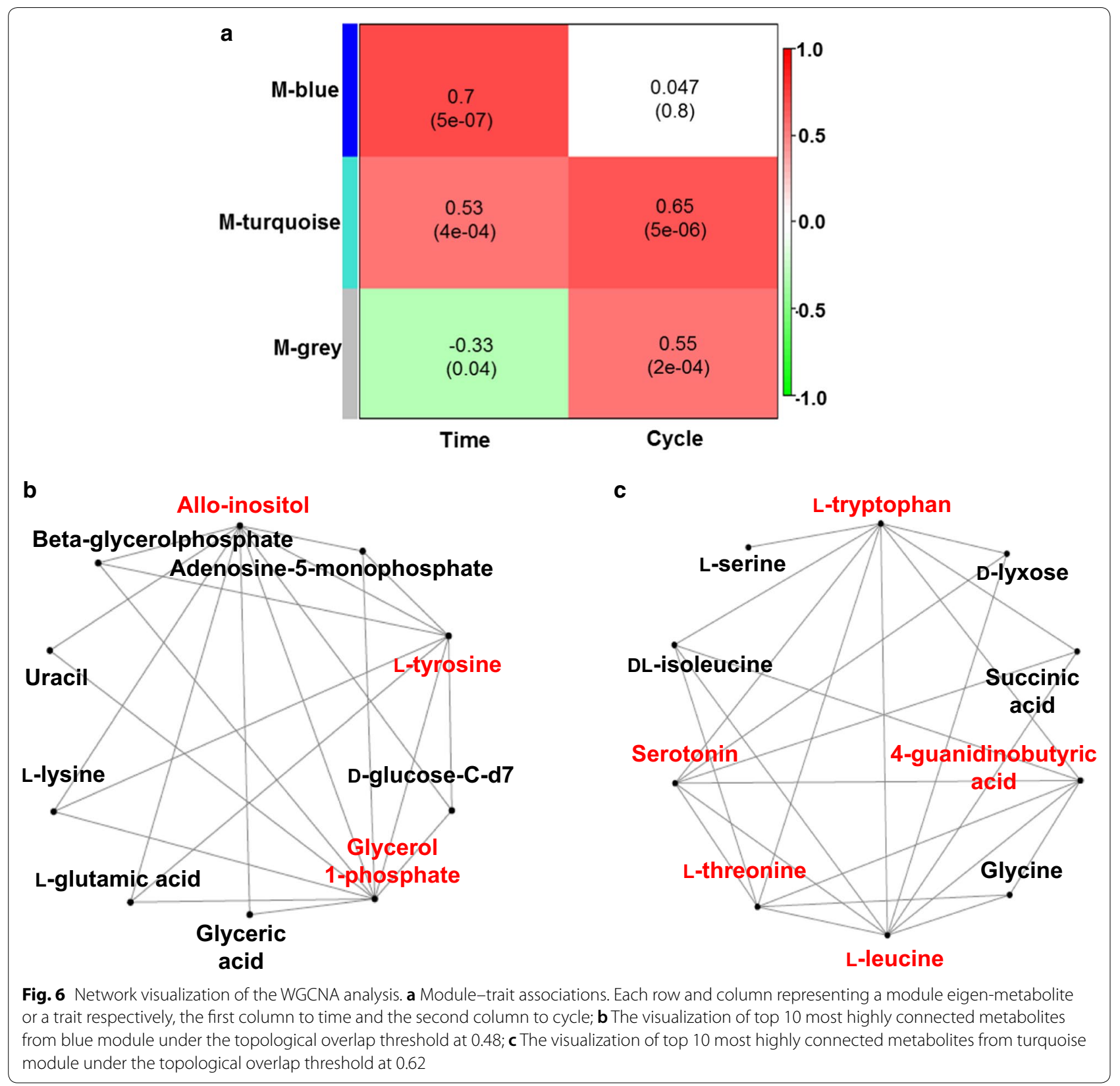

decreased nitrogen utilization capacity, and continuous down-regulated glycolysis and TCA cycle were correlated with the decreased stability of culture system. At last, some biomarkers, such as Pyr, Cit, OXA, FUM, L-tryptophan, L-threonine, L-leucine, serotonin, and 4-guanidinobutyric acid, correlated with the stability of the culture system of C. cohnii M-1-2 were identified. This study provided not only an efficient and energy-saving strategy for industrial production of DHA, but also important information at metabolite level to monitor the stability of culture system in C. cohnii during the long-term repeated fed-batch fermentation. This strategy could also be useful for cultivating other heterotrophic algae to improve productivity and reduce production cost. Moreover, the revealed mechanisms were useful to further optimize production processes for $C$. cohnii and other heterotrophic algae during repeated fed-batch cultivation.

\section{Methods}

\section{Microorganism and medium}

Crypthecodinium cohnii M-1-2, a mutant of C. cohnii ATCC 30556, was previously isolated by our laboratory 
[44]. C. cohnii M-1-2 is maintained in the basal medium containing $25 \mathrm{~g} / \mathrm{L}$ sea salt (Sigma-Aldrich, USA), $2 \mathrm{~g} / \mathrm{L}$ yeast extract (OXOID, UK), and $9 \mathrm{~g} / \mathrm{L}$ glucose monohydrate (Jiang Tian, China) [20]. The typical fermentation medium includes $25 \mathrm{~g} / \mathrm{L}$ sea salt, $6 \mathrm{~g} / \mathrm{L}$ yeast extract, and $27 \mathrm{~g} / \mathrm{L}$ glucose monohydrate [44].

\section{Repeated fed-batch culture}

The seed culture was prepared as follows: C. cohnii M-1-2 was inoculated into basal medium, and cultivated for $48 \mathrm{~h}$ at $25^{\circ} \mathrm{C}$ and $180 \mathrm{rpm}$; then, $10 \%(\mathrm{v} / \mathrm{v})$ cultures were inoculated into fermentation medium and cultivated for $84 \mathrm{~h}$ at $25^{\circ} \mathrm{C}$ and $180 \mathrm{rpm}$. The method to establish individual fermentation cycle was according to our previous publication with minor modification [3]. Briefly, all fed-batch cultures were carried out in a 5-L fermenter (Demei, China); the dissolved oxygen was maintained above $30 \%$ air saturation with $1 \mathrm{vvm}$ of the air flow level, and automatic regulation of agitation speed; the cultivation temperature was maintained at $25 \pm 0.2{ }^{\circ} \mathrm{C}$; and the $\mathrm{pH}$ was maintained at $6.5 \pm 0.1$ with $0.5 \mathrm{M} \mathrm{H}_{2} \mathrm{SO}_{4}$. The antifoam SE-15 was added to eliminate foam when needed. The $50 \%(w / v)$ yeast extract was used as supplementary nitrogen source between 12 and $96 \mathrm{~h}$ of each cycle.

For repeated fed-batch culture, 70-90\% of the fermentation broth was removed at $120 \mathrm{~h}$ of each cycle and then the same volume of fresh medium was supplemented. $10-30 \%$ of the fermentation broth left from the previous fed-batch culture was used as seeds for the next fedbatch culture. To optimize glucose concentration during the fermentation, the initial inoculation volume was $10 \%$ $(v / v)$ and the replacement ratio of the medium was $90 \%$ $(v / v)$, respectively, and the glucose concentrations were maintained at $5-15 \mathrm{~g} / \mathrm{L}, 15-27 \mathrm{~g} / \mathrm{L}$ or $27-45 \mathrm{~g} / \mathrm{L}$ by feeding with $80 \%(w / v)$ glucose monohydrate solution. To optimize the replacement ratio, the initial inoculation volume was selected as $10 \%(v / v)$ with the initial glucose monohydrate concentration $27 \mathrm{~g} / \mathrm{L}$, and glucose concentration was maintained at $15-27 \mathrm{~g} / \mathrm{L}$. Replacement ratios of 70,80 and $90 \%(v / v)$ were evaluated.

\section{Analysis of DCW, glucose concentration, total lipid content and fatty acids profile}

The DCW was measured by gravimetric method after freeze-drying of C. cohnii cells [3]. The glucose concentration was measured with a Glucose Assay Kit (Biosino, China) $[3,22]$. Total lipid content was extracted according to the previous study with a minor modification [45]. Briefly, $25 \mathrm{mg}$ freeze-dried cells of C. cohnii was extracted by chloroform:methanol $(2: 1, v / v)$ adding $0.01 \%$ butylated hydroxytoluene for three times, and then was washed by $1 \mathrm{M} \mathrm{KCl}$ solution and $\mathrm{ddH}_{2} \mathrm{O}$ for one time, respectively. Fatty acids profile were measured according to the previous study [40,46]. Briefly, freeze-dried cells of $C$. cohnii adding with heptadecanoic acid methanol solution (an internal standard), methanol of 3\% $(v / v)$ sulfuric acid, and chloroform were conducted for reaction at $100{ }^{\circ} \mathrm{C}$ for $4 \mathrm{~h}$. The chloroform phase was used to detect after stratification by $\mathrm{ddH}_{2} \mathrm{O}$.

\section{LC- and GC-MS based metabolomic analysis}

Cell samples collected at $60 \mathrm{~h}$ and $96 \mathrm{~h}$ for each of the four fermentation cycles of the repeated fed-batch culture were used for LC- and GC-MS metabolomics analyses according to the previous publication [20]. Three and five biological replicates were carried out for LC- and GC-MS metabolomics analysis respectively. The data processing for $\mathrm{LC}-$ and GC-MS metabolomics analyses was conducted following the previous study [20]. Metabolomic data was normalized by both cells number and interior control. For LC-MS metabolomic analysis, all standards were purchased from Sigma-Aldrich (MO, USA), and the heatmap was created using MeV 4.9.0 software. For GC-MS metabolomic analysis, principal component analysis (PCA) was created by MetaboAnalyst 4.0 software available at https://www.metaboanalyst.ca/.

\section{Weighted gene co-expression network analysis (WGCNA)} WGCNA was carried out for GC-MS metabolomic analysis. The method for network construction, module identification, and the relationship between modules and external conditions/traits followed the standard metabolomic analysis procedure of WGCNA described previously [37]. All metabolomic analyses were firstly converted into z-score for WGCNA construction. To identify the biologically significant modules with cycle and time through WGCNA, the value of the correlation coefficient $r$ was set more than 0.6 and the statistical confidence $p$-value was set less than 0.05 [20]. Further downstream analysis for associated modules of biological significance was carried out to identify potential hub metabolites. Visualization of 10 most highly connected metabolites was conducted using the Cytoscape software [47]. Metabolites with a number of connections greater than or equal to 5 with other metabolites were considered as hub metabolites [20].

\section{Statistical analysis}

All measurements of DCW, glucose concentration, total lipid content and fatty acids profile were performed in at least three analytical replicates. All data were expressed in the form of mean values \pm standard deviations. Student's $T$-test was used to evaluate the data (" $p<0.05$, "** $\left.p<0.01,{ }^{* * * *} p<0.001\right)$. 


\begin{abstract}
Abbreviations
DHA: Docosahexaenoic acid; DCW: Dry cell weight; PUFAs: Polyunsaturated fatty acids; TFAs: Total fatty acids; EPA: Eicosapentaenoic acid; ARA: Arachidonic acid; PCA: Principal component analysis; TCA: Tricarboxylic acid; ATP: Adenosine triphosphate; F6P: Fructose 6-phosphate; Suc: Succinate; ADP-Glu: Adenosine diphosphoglucose; R5P: Ribose 5-phosphate; 1,3-PG: 1,3-Bisphosphoglyceric acid; NAD: Nicotinamide adenine dinucleotide; Mal: Malate; ADP Adenosine diphosphate; Asp: Aspartic acid; GAP: Glyceraldehyde 3-phosphate; Glu: Glutamic acid; AMP: Adenosine monophosphate; Phe: Phenylalanine; NADPH: Nicotinamide adenine dinucleotide phosphate; RiBP: Ribulose 1,5-bisphosphate; 3-PG: 3-Phosphoglyceric acid; PEP: Phosphoenolpyruvic acid; NADP: Oxidized form of nicotinamide adenine dinucleotide phosphate; AKG: 2-Oxoglutaric acid; CoA: Coenzyme A; FUM: Fumaric acid; OXA: Oxaloacetic acid; Cit: Citric acid; Pyr: Pyruvate; AcCoA: Acetyl coenzyme A; FBP: Fructose 1,6-bisphosphate.
\end{abstract}

\section{Acknowledgements}

Not applicable.

\section{Authors' contributions}

$L L$ and WZ conceived the project and designed the experiments. $L L, J C$, JD and ML performed the experiments. LL and GP analyzed the data. LL wrote the manuscript. LL, FW, LC and WZ revised the manuscript. All authors read and approved the final manuscript.

\section{Funding}

This research was supported by grants from the National Natural Science Foundation of China (Nos. 31770100, 31972931, 31770035, 91751102, 21621004, 31370115 and 31470217), the National Key Research and Development Program of China (Nos. 2019YFA0904600, 2018YFA0903000 and 2018YFA0903600), Tianjin Synthetic Biotechnology Innovation Capacity Improvement Project (No. TSBICIP-KJGG-007), Tianjin Municipal Science Foundation (No. 18JCQNJC10000) and Zaoneng Biotechnology Inc.

\section{Availability of data and materials}

The datasets used and analyzed during the current study are available from the corresponding author on reasonable request.

\section{Ethics approval and consent to participation}

Not applicable.

\section{Consent for publication}

Not applicable.

\section{Competing interests}

The authors declare that they have no competing interests.

\section{Author details}

${ }^{1}$ Laboratory of Synthetic Microbiology, School of Chemical Engineering \& Technology, Tianjin University, Tianjin 300072, People's Republic of China.

${ }^{2}$ Frontier Science Center for Synthetic Biology and Key Laboratory of Systems Bioengineering (MOE), School of Chemical Engineering and Technology, Tianjin University, Tianjin 300350, People's Republic of China. ${ }^{3}$ Center for Biosafety Research and Strategy, Tianjin University, Tianjin, People's Republic of China. ${ }^{4}$ SynBio Research Platform, Collaborative Innovation Center of Chemical Science and Engineering (Tianjin), Tianjin, People's Republic of China.

Received: 28 November 2019 Accepted: 8 April 2020

Published online: 16 April 2020

\section{References}

1. Muskiet FA, Fokkema MR, Schaafsma A, Boersma ER, Crawford MA. Is docosahexaenoic acid (DHA) essential? Lessons from DHA status regulation, our ancient diet, epidemiology and randomized controlled trials. J Nutr. 2004;134:183-6.

2. Horrocks LA, Yeo YK. Health benefits of docosahexaenoic acid (DHA). Pharmacol Res. 1999;40:211-25.

3. Liu L, Wang F, Yang J, Li X, Cui J, Liu J, Shi M, Wang K, Chen L, Zhang W. Nitrogen feeding strategies and metabolomic analysis to alleviate high-nitrogen inhibition on docosahexaenoic acid production in Crypthecodinium cohnii. J Agric Food Chem. 2018;66:10640-50.

4. Guo DS, Ji XJ, Ren LJ, Li GL, Huang H. Improving docosahexaenoic acid production by Schizochytrium sp. using a newly designed high-oxygensupply bioreactor. AICHE J. 2017:63:4278-86.

5. Diao J, Song X, Cui J, Liu L, Shi M, Wang F, Zhang W. Rewiring metabolic network by chemical modulator based laboratory evolution doubles lipid production in Crypthecodinium cohnii. Metab Eng. 2018;51:88-98.

6. Mendes A, Reis A, Vasconcelos R, Guerra P, Lopes da Silva T. Crypthecodinium cohnii with emphasis on DHA production: a review. J Appl Phycol. 2008;21:199-214.

7. Rumiani LA, Jalili H, Amrane A. Enhanced docosahexaenoic acid production by Crypthecodinium cohnii under combined stress in two-stage cultivation with date syrup based medium. Algal Res. 2018;34:75-81.

8. De Swaaf ME, Sijtsma L, Pronk JT. High-cell-density fed-batch cultivation of the docosahexaenoic acid producing marine alga Crypthecodinium cohnii. Biotechnol Bioeng. 2003;81:666-72.

9. De Swaaf ME, Pronk JT, Sijtsma L. Fed-batch cultivation of the docosahexaenoic-acid-producing marine alga Crypthecodinium cohnii on ethanol. Appl Microbiol Biotechnol. 2003;61:40-3.

10. Giridhar R, Srivastava AK. Repeated fed-batch sorbose fermentation by Gluconobacter oxydans. Chem Biochem Eng Q. 2001;15:127-9.

11. Ohya T, Ohyama M, Kobayashi K. Optimization of human serum albumin production in methylotrophic yeast Pichia pastoris by repeated fed-batch fermentation. Biotechnol Bioeng. 2005;90:876-87.

12. Qu L, Ren LJ, Sun GN, Ji XJ, Nie ZK, Huang H. Batch, fed-batch and repeated fed-batch fermentation processes of the marine thraustochytrid Schizochytrium sp. for producing docosahexaenoic acid. Bioprocess Biosyst Eng. 2013;36:1905-12.

13. Xue X, Li W, Li Z, Xia Y, Ye Q. Enhanced 1,3-propanediol production by supply of organic acids and repeated fed-batch culture. J Ind Microbiol Biotechnol. 2010;37:681-7.

14. Ji XJ, Zhang AH, Nie ZK, Wu WJ, Ren LJ, Huang H. Efficient arachidonic acid-rich oil production by Mortierella alpina through a repeated fedbatch fermentation strategy. Bioresour Technol. 2014;170:356-60.

15. Han X, Song W, Liu G, Li Z, Yang P, Qu Y. Improving cellulase productivity of Penicillium oxalicum RE-10 by repeated fed-batch fermentation strategy. Bioresour Technol. 2017;227:155-63.

16. Xie YP, Ho SH, Chen CY, Chen CNN, Liu CC, Ng IS, Jing KJ, Yang SC, Chen $\mathrm{CH}$, Chang JS, Lu YH. Simultaneous enhancement of $\mathrm{CO}_{2}$ fixation and lutein production with thermo-tolerant Desmodesmus sp. F51 using a repeated fed-batch cultivation strategy. Biochem Eng J. 2014;86:33-40.

17. Zhao X, Hu C, Wu S, Shen H, Zhao ZK. Lipid production by Rhodosporidium toruloides $Y 4$ using different substrate feeding strategies. J Ind Microbiol Biotechnol. 2011;38:627-32.

18. Weckwerth W. Metabolomics in systems biology. Annu Rev Plant Biol. 2003;54:669-89.

19. Marta C, Silvia M. Metabolomics and fluxomics approaches. Essays Biochem. 2008:45:67-81.

20. Sui X, Niu X, Shi M, Pei G, Li J, Chen L, Wang J, Zhang W. Metabolomic analysis reveals mechanism of antioxidant butylated hydroxyanisole on lipid accumulation in Crypthecodinium cohnii. J Agric Food Chem. 2014;62:12477-84.

21. Li J, Niu X, Pei G, Sui X, Zhang X, Chen L, Zhang W. Identification and metabolomic analysis of chemical modulators for lipid accumulation in Crypthecodinium cohnii. Bioresour Technol. 2015;191:362-8.

22. Li X, Pei G, Liu L, Chen L, Zhang W. Metabolomic analysis and lipid accumulation in a glucose tolerant Crypthecodinium cohnii strain obtained by adaptive laboratory evolution. Bioresour Technol. 2017;235:87-95.

23. Jiang $Y$, Chen F. Effects of medium glucose concentration and $\mathrm{pH}$ on docosahexaenoic acid content of heterotrophic Crypthecodinium cohnii. Process Biochem. 2000;35:1205-9.

24. De Swaaf ME, De Rijk TC, Eggink G, Sijtsma L. Optimisation of docosahexaenoic acid production in batch cultivations by Crypthecodinium cohnii. J Biotechnol. 1999;70:185-92.

25. Ozmihci S, Kargi F. Ethanol fermentation of cheese whey powder solution by repeated fed-batch operation. Enzyme Microb Technol. 2007:41:169-74.

26. Cornet I, Van Derlinden E, Cappuyns AM, Van Impe JF. Heat stress adaptation of Escherichia coli under dynamic conditions: effect of inoculum size. Lett Appl Microbiol. 2010;51:450-5. 
27. Swaaf M, Grobben G, Eggink G, Rijk T, Meer P, Sijtsma L. Characterisation of extracellular polysaccharides produced by Crypthecodinium cohnii. Appl Microbiol Biotechnol. 2001;57:395-400.

28. Ito T, Sota H, Honda H, Shimizu K, Kobayashi T. Efficient acetic acid production by repeated fed-batch fermentation using two fermentors. Appl Microbiol Biotechnol. 1991;36:295-9.

29. Matsudo MC, Bezerra RP, Sato S, Perego P, Converti A, Carvalho JCM. Repeated fed-batch cultivation of Arthrospira (Spirulina) platensis using urea as nitrogen source. BiochemEng J. 2009:43:52-7.

30. Sirisansaneeyakul S, Wannawilai S, Chisti Y. Repeated fed-batch production of xylitol by Candida magnoliae TISTR 5663. J Chem Technol Biotechnol. 2013;88:1121-9.

31. Moeller L, Grunberg M, Zehnsdorf A, Aurich A, Bley T, Strehlitz B. Repeated fed-batch fermentation using biosensor online control for citric acid production by Yarrowia lipolytica. J Biotechnol. 2011;153:133-7.

32. Furlan SA, Delia-Dupuy ML, Strehaiano P. Xylitol production in repeated fed batch cultivation. World J Microbiol Biotechnol. 1997;13:591-2.

33. Zelic B, Gostovic S, Vuorilehto K, Vasic-Racki D, Takors R. Process strategies to enhance pyruvate production with recombinant Escherichia coli: from repetitive fed-batch to in situ product recovery with fully integrated electrodialysis. Biotechnol Bioeng. 2004;85:638-46.

34. Sato S, Yanagisawa S. Characterization of metabolic states of Arabidopsis thaliana under diverse carbon and nitrogen nutrient conditions via targeted metabolomic analysis. Plant Cell Physiol. 2014;55:306-19.

35. Jarak I, Almeida S, Carvalho RA, Sousa M, Barros A, Alves MG, Oliveira PF. Senescence and declining reproductive potential: insight into molecular mechanisms through testicular metabolomics. Biochim Biophys Acta Mol Basis Dis. 2018;1864:3388-96.

36. Zhou YZ, Yan ML, Gao L, Zhang JQ, Qin XM, Zhang X, Du GH. Metabonomics approach to assessing the metabolism variation and gender gap of Drosophila melanogaster in aging process. Exp Gerontol. 2017:98:110-9.

37. Pei G, Chen L, Zhang W. WGCNA application to proteomic and metabolomic data analysis. Methods Enzymol. 2017;585:135-58.

38. Horvath S, Dong J. Geometric interpretation of gene coexpression network analysis. PLoS Comput Biol. 2008:4:e1000117.

39. Henderson RJ, Leftley JW, Sargent JR. Lipid composition and biosynthesis in the marine dinoflagellate Crypthecodinium cohnii. Phytochemistry. 1988;27:1679-83.

40. Pei G, Li X, Liu L, Liu J, Wang F, Chen L, Zhang W. De novo transcriptomic and metabolomic analysis of docosahexaenoic acid (DHA)-producing
Crypthecodinium cohnii during fed-batch fermentation. Algal Res. 2017;26:380-91.

41. Balazadeh S, Schildhauer J, Araujo WL, Munne-Bosch S, Fernie AR, Proost $\mathrm{S}$, Humbeck K, Mueller-Roeber B. Reversal of senescence by N resupply to N-starved Arabidopsis thaliana: transcriptomic and metabolomic consequences. J Exp Bot. 2014;65:3975-92.

42. Xiu ZL, Zeng AP, Deckwer WD. Model analysis concerning the effects of growth rate and intracellular tryptophan level on the stability and dynamics of tryptophan biosynthesis in bacteria. J Biotechnol. 1997;58:125-40.

43. Diao J, Song X, Zhang X, Chen L, Zhang W. Genetic engineering of Crypthecodinium cohnii to increase growth and lipid accumulation. Front Microbiol. 2018:9:492.

44. Liu J, Pei G, Diao J, Chen Z, Liu L, Chen L, Zhang W. Screening and transcriptomic analysis of Crypthecodinium cohnii mutants with high growth and lipid content using the acetyl-CoA carboxylase inhibitor sethoxydim. Appl Microbiol Biotechnol. 2017;101:6179-91.

45. Yang S, Lu SH, Yuan YJ. Cerium elicitor-induced phosphatidic acid triggers apoptotic signaling development in Taxus cuspidata cell suspension cultures. Chem Phys Lipids. 2009;159:13-20.

46. Wei X, Xiufeng L, Jinyi X, Qingyu W. High-density fermentation of microalga Chlorella protothecoides in bioreactor for microbio-diesel production. Appl Microbiol Biotechnol. 2008;78:29-36.

47. Shannon P, Markiel A, Ozier O, Baliga NS, Wang JT, Ramage D, Amin N, Schwikowski B, Ideker T. Cytoscape: a software environment for integrated models of biomolecular interaction networks. Genome Res. 2003;13:2498-504.

48. Da Silva TL, Mendes A, Mendes RL, Calado V, Alves SS, Vasconcelos JM, Reis A. Effect of n-dodecane on Crypthecodinium cohnii fermentations and DHA production. J Ind Microbiol Biotechnol. 2006;33:408-16.

49. Mendes A, Guerra P, Madeira V, Ruano F, Lopes da Silva T, Reis A. Study of docosahexaenoic acid production by the heterotrophic microalga Crypthecodinium cohnii CCMP 316 using carob pulp as a promising carbon source. World J Microbiol Biotechnol. 2007;23:1209-15.

\section{Publisher's Note}

Springer Nature remains neutral with regard to jurisdictional claims in published maps and institutional affiliations.
Ready to submit your research? Choose BMC and benefit from:

- fast, convenient online submission

- thorough peer review by experienced researchers in your field

- rapid publication on acceptance

- support for research data, including large and complex data types

- gold Open Access which fosters wider collaboration and increased citations

- maximum visibility for your research: over $100 \mathrm{M}$ website views per year

At BMC, research is always in progress.

Learn more biomedcentral.com/submissions 